\title{
EFECTOS DEL GASTO PÚBLICO SOBRE LA PRODUCCIÓN DE LOS ESTADOS DE MÉXICO, 2004-2018: UN ANÁLISIS DE PANEL DE DATOS ESPACIAL^^
}

\author{
EFFECTS OF PUBLIC EXPENDITURE ON THE GROSS \\ DOMESTIC PRODUCT OF THE STATES OF MEXICO, 2004- \\ 2019: A SPATIAL DATA PANEL ANALYSIS \\ Pablo Mejía Reyes* \\ Liliana Rendón Rojas** \\ Marlen Rocío Reyes Hernández*** \\ Roberto Ramírez Rodríguez****
}

enviado: 24 marzo 2021 - aceptado: 02 junio 2021

\section{Resumen}

En este trabajo se analizan los efectos del gasto público, corriente y de inversión, sobre el producto interno bruto (PIB) en los estados de México durante el período 2004-2018. Para ello se estiman modelos econométricos espaciales para datos de panel, controlando por el impacto de otros determinantes. Los resultados sugieren la existencia de interacciones espaciales entre las tasas de crecimiento del PIB,

\footnotetext{
Mejía Reyes, P., Rendón Rojas, L., Reyes Hernández, M. R., \& Ramírez Rodríguez, R. (2022). Efectos del gasto público sobre la producción de los estados de México, 2004-2018: un análisis de panel de datos espacial. Estudios económicos, 39(78), pp. 97-125. DOI: https://doi.org/10.52292/j. estudecon.2022.2568

* Centro de Investigación en Ciencias Económicas, Facultad de Economía, Universidad Autónoma del Estado de México, México. ORCID: orcid.org/0000-0002-9222-1526. Correo electrónico: pmejiare@uaemex.mx

** Centro de Investigación en Ciencias Económicas, Facultad de Economía, Universidad Autónoma del Estado de México, México. ORCID: orcid.org/0000-0003-1735-8757. Correo electrónico. Irendonr@uaemex.mx

*** Centro de Investigación en Ciencias Económicas, Facultad de Economía, Universidad Autónoma del Estado de México, México. ORCID: orcid.org/0000-0002-8339-4011. Correo electrónico: mrreyesh@uaemex.mx

**** Departamento de Economía, Universidad de Sonora, México. ORCID: orcid.org/0000-0001-71038469. Correo electrónico: roberto.ramirez@unison.mx
} 
así como efectos positivos del crecimiento del sector productor de bienes comerciables y la especialización de la planta productiva, pero negativos (hipótesis de suavizamiento) de la razón de remesas a PIB. Interesantemente, no hay evidencia de efectos significativos de ningún tipo de gasto público, lo que implica serias limitaciones de los gobiernos estatales para impulsar la producción.

Palabras clave: gasto público, producto interno bruto, modelos econométricos espaciales para datos de panel, estados de México.

Códigos JEL: C33, E62, H53, H54, O47.

\begin{abstract}
This paper analyses the effects of public current and investment expenditure on the gross domestic product (GDP) growth in the states of Mexico over the period 20042018. To do this, econometric spatial panel data models are estimated, controlling for the impact of other determinants. The results suggest the existence of spatial interactions between GDP growth rates, as well as positive effects of growth of the tradable goods producing sector and the specialization of the production structure, but negative of the remittance to GDP ratio (smoothing hypothesis). Interestingly, there is no evidence of significant effects of any kind of public spending, implying serious limitations of state governments in boosting production.
\end{abstract}

Keywords: public expenditure, gross domestic product, econometric spatial panel data models, Mexican states.

JEL Codes: C33, E62, H53, H54, O47. 


\section{INTRODUCCIÓN}

El análisis del impacto del gasto público sobre la producción ha estado sujeto a un amplio y largo debate tanto desde un punto de vista teórico como empírico. Aunque los efectos de largo plazo del gasto total pueden ser ambiguos (Arslanalp, Bornhorst \& Gupta, 2011; Pinilla, Jiménez \& Montero, 2013; Bagdigen \& Cetintas, 2004), invariablemente se concluye que la inversión pública tiene efectos positivos cuando se destina a la creación de infraestructura porque complementa la inversión privada (Barro, 1990 y Barro \& Sala-i-Martin, 1995). Ha existido, sin embargo, un largo debate sobre sus efectos de corto y mediano plazos debido a que, por un lado, afecta positivamente a la demanda agregada, lo que contribuye a expandir la producción, pero, por otro, puede desplazar a la inversión privada por sus efectos sobre la tasa de interés o por las distorsiones que pueden derivarse de la creación de impuestos adicionales para financiarlo (Lindbeck, 1983; King $\&$ Rebelo, 1990). Más aún, otros autores concluyen que la presencia excesiva del sector público puede obstruir la promoción del crecimiento en una economía abierta cuando su financiamiento con deuda presiona la tasa de interés al alza, lo que impulsa la entrada de capitales y aprecia el tipo de cambio real, provocando, a su vez, un desplazamiento de las exportaciones netas (Hernández \& Velázquez, 2019).

Por lo anterior, se sigue que un consenso sobre los efectos del gasto público en el producto está lejos de alcanzarse y que los resultados empíricos dependen de la especificación del modelo, el método estadístico de análisis, la información de gasto público utilizada y la calidad de los datos, entre otros factores. En este marco, el objetivo del presente documento es reevaluar los efectos del gasto público, corriente y de inversión, sobre el crecimiento de la producción en los 32 estados de México, considerando la posibilidad de interacción espacial y controlando por los efectos de otras variables relevantes, durante el período 2004-2018. La estimación de modelos espaciales para datos de panel sugiere que el crecimiento de la producción depende principalmente de los efectos de las variables de control de la misma unidad espacial, y en menor medida de lo que acontece en la vecindad, especialmente de la producción de bienes comerciables y de la especialización. Interesantemente, los resultados muestran que el gasto público no tiene efectos significativos sobre la producción.

El resto de este documento está conformado por cuatro secciones adicionales. En la primera se discute la literatura con mayor detalle, enfatizando el caso de los estados de México, en tanto que en la segunda se presenta el modelo espacial de panel de datos a estimar y la metodología para seleccionar la mejor especificación. En la tercera sección se exponen y discuten los resultados de la estimación econométrica, y en la última se establecen las conclusiones principales. 


\section{REVISIÓN DE LITERATURA}

La ambigüedad de los efectos del gasto público sobre la producción, tanto a nivel teórico como empírico, ha llevado a los investigadores a buscar diferencias en función del horizonte de tiempo, su composición y su tamaño.

Desde la perspectiva de la nueva economía keynesiana (NEK) ${ }^{1}$, un incremento temporal del gasto público eleva la demanda de bienes y disminuye el ahorro nacional deseado. En ese sentido, el aumento de las compras del Estado solo afecta a la producción elevando la demanda agregada, por lo que el tipo de política de gasto descrita anteriormente se considera expansiva, y viceversa. Más todavía, esta posibilidad da pie a que la política fiscal pueda utilizarse como instrumento de estabilización para contrarrestar la magnitud de las fluctuaciones cíclicas, especialmente durante las recesiones cuando la eficacia de la política monetaria es limitada y el recorte de los salarios puede no ser efectivo ante la posibilidad de que se reduzca adicionalmente la demanda agregada. Desde esta perspectiva, entonces, la política fiscal podría ser contracíclica ${ }^{2}$.

Existe la posibilidad, sin embargo, de que el gasto público afecte mínima o negativamente al producto debido al "desplazamiento" de inversión privada que resulta del incremento de la tasa de interés que exige el financiamiento público. Más aún, el impacto multiplicador del gasto podría no ser tan alto debido a particularidades en torno a la aplicación del gasto, tales como la forma de financiarlo (efecto distorsionante de los impuestos), la incertidumbre macroeconómica, especialmente en fases recesivas, y el grado de apertura comercial, que puede llevar a la apreciación cambiaria, con efectos negativos sobre las exportaciones netas (Hansson, 1984; Lindbeck, 1983).

La tradición neoclásica, por su lado, sostiene que el gasto público solo podría incidir en el crecimiento económico a largo plazo cuando se destina a incrementar la productividad del capital y del trabajo a través de la dotación de infraestructura institucional, productiva, tecnológica, académica y de salud, entre otras (Engen \& Skinner, 1992) $)^{3}$. Por lo contario, el gasto corriente puede tener un efecto neutro o negativo sobre el crecimiento ya que no incrementa el patrimonio público

\footnotetext{
Véanse Greenwald, \& Stiglitz (1987) y Mankiw (1989) para una visión más detallada.

Véase Sorensen \& Whitta-Jacobsen (2010) para una exposición formal.

En otras palabras, el Estado propicia la creación de bienes públicos que el sector privado no estaría dispuesto a producir debido a que no son rentables, pero que son necesarios para la sociedad.
} 
federal pues la mayor parte se destina a sueldos y salarios y programas sociales (Barro \& Sala-i-Martin, 1992; Turnovsky, 1996) ${ }^{4}$.

Los estudios a nivel internacional sobre los efectos del gasto público en el crecimiento del producto han reportado evidencia mixta o con un impacto positivo solo de componentes específicos (Olugbenga \& Owoye, 2007; Pinilla, Jiménez \& Montero, 2013). Los análisis para países específicos reflejan la misma problemática. En particular, Bagdigen y Cetintas (2004) analizan el caso de Turquía y no hallan una relación significativa entre gasto público y crecimiento, en tanto que Nworji, Okwn, Obiwurn y Nworji, (2012) sugieren que los gastos ordinarios en servicios económicos ejercen un efecto negativo, pero no significativo, y el gasto en transferencias tiene un efecto positivo y significativo. Por el contrario, Posada y Gómez (2002) analizan el tamaño óptimo del gasto público y argumentan que este tiene un efecto positivo en la economía colombiana. Del mismo modo, Arslanalp, Bornhorst y Gupta (2011) argumentan que el capital público tiene una relación positiva con el crecimiento que varía con el nivel de capital público ${ }^{5}$.

En el caso de México, se ha argumentado que durante las últimas cuatro décadas se ha transitado por una senda de lento crecimiento (Moreno-Brid \& Ros, 2009; Lustig, 2002) y que su determinante próximo es la baja tasa de inversión en capital físico, cuya reducción se ha debido, en buena medida, a una notable contracción de la inversión pública (Ros, 2015; Fonseca, 2009). Los estudios más específicos existentes han utilizado indicadores de gasto público o han construido indicadores de infraestructura física. Por ejemplo, Hernández (2011) sostiene que no existe correlación entre inversión pública y crecimiento económico durante el período 1980-2009, y que la existencia de autocorrelación de los errores de los modelos estimados sugiere que la inversión pública y privada no son los determinantes fundamentales de largo plazo, sino que son las deficiencias estructurales de la economía. Por su parte, Noriega y Fontenla (2007) sugieren que, entre 1950 y 2006, los choques en infraestructura de electricidad y caminos tienen efectos positivos en la producción, aunque no existe causalidad en el sentido de Granger para ningún par de variables, y que los choques a la tendencia de la inversión en telefonía no tienen un efecto permanente en la producción real a largo plazo.

4 Otros trabajos han estudiado los efectos del gasto público clasificándolo como productivo e improductivo o financiado con impuestos distorsionantes y no distorsionantes. Generalmente se pronostica que el gasto productivo financiado con impuestos no distorsionantes tendrá un efecto positivo sobre el crecimiento de largo plazo, y viceversa (Semmler, Greiner, Diallo, Rajaram \& Rezai, 2011).

5 En países con niveles menores al $60 \%$ del PIB, cada unidad adicional de capital público tiene el máximo efecto sobre el crecimiento. 
A nivel regional ${ }^{6}$, son pocos los trabajos que analizan el efecto de los distintos rubros del gasto público en el crecimiento de los estados de México, aunque hay algunos que abordan la influencia del gasto en inversión, en general, y de diferentes indicadores de infraestructura física, en particular, tanto para los 32 estados del país como para algunas regiones particulares. En relación con la evidencia para los 32 estados que conforman México, Sánchez y García (2016) estiman modelos dinámicos de datos de panel con información de 1993 a 2012 y muestran que la deuda pública se correlaciona positivamente con la inversión pública y que esta, a su vez, genera crecimiento económico 7 . De igual forma, Srithongrung y Sánchez (2015), mediante un modelo de corrección de error, muestran que los efectos de la inversión pública sobre el crecimiento son estadísticamente significativos y positivos tanto a corto como a largo plazo durante el período de 1993 a $2011^{8}$.

En contraste, Díaz, Mejía, Reyes y Desiderio (2018) muestran que si bien el gasto público total ha sido relevante en la explicación del crecimiento económico estatal, sobre todo en aquellos estados más grandes del país, los gastos que realizan los gobiernos estatales en materia de inversión no tienen un efecto significativo en el crecimiento económico para el período de 1999 a 20149. Por su parte, Fuentes (2003) sostiene que su indicador de infraestructura tiene efectos estadísticamente significativos en la explicación de las variaciones regionales del $\mathrm{PIB}^{10}$, mientras que Fuentes y Mendoza (2003) analizan el papel de las características y la magnitud de la dotación de infraestructura pública por regiones en el proceso de convergencia; encuentran que entre 1980 y 1985 la relación capital público-PIB es positiva y significativa, pero que esa relación desaparece entre 1985 y 1998, lo que pudo

6 A nivel internacional, por ejemplo, Bajo y Díaz (2003) muestran que el capital público y las transferencias tienen un efecto positivo sobre el crecimiento de las regiones españolas (1967-1995), siendo relativamente más intenso el del primero en las regiones más productivas y el del segundo en las menos productivas. Por su parte, Marjit, Sasmal y Sasmal (2013), para los estados de la India, muestran que los gastos corrientes tienen un efecto negativo sobre el crecimiento, mientras que los gastos en infraestructura, energía, comunicaciones y transporte tienen un impacto significativo y positivo en el crecimiento, mientras que los gastos en salud y educación no tienen efectos significativos.

7 Sánchez y García (2016) consideran como variables de control a la población, años promedio de escolaridad e inversión extranjera directa.

8 Srithongrung y Sánchez (2015) consideran como variables de control la tasa de crecimiento anual de la calidad del trabajo medido por los cambios en el nivel promedio de educación, la tasa de crecimiento anual de la población y el cambio anual en la inversión privada.

9 Utilizan regresión lineal y regresiones cuantílica y decílica; el empleo es considerado como una variable de control.

10 Reporta, además, la existencia de grandes disparidades en la dotación de infraestructura y en sus diferentes componentes. 
deberse al cambio de prioridades del gobierno, entonces centrado en la estabilidad macroeconómica ${ }^{11}$.

Entre los análisis para regiones más específicas, está el trabajo de Mejía, Reyes y Sánchez (2017), quienes analizan la relación entre diferentes componentes del gasto público y el PIB per cápita mediante análisis de largo plazo con cambio estructural para ocho estados del centro de México ${ }^{12}$ en el período 1980-2012; sus resultados muestran que solo en tres estados existen relaciones de largo plazo entre las variables analizadas y que en los otros se limitan a pocas variables y períodos. Para la misma muestra, pero con referencia a la relación entre los componentes del gasto público y el ciclo económico, Mejía y Sánchez (2019) argumentan que la política fiscal nacional fue procíclica entre 1980 y finales de los años noventa, pero que a partir de entonces sus efectos no han sido estadísticamente significativos. El análisis por estados refleja relaciones cambiantes y poco robustas, que podrían explicar la alta discrecionalidad en el manejo del gasto público estatal.

Por último, Barajas y Gutiérrez (2012) analizan el papel de la infraestructura social ${ }^{13}$ y económica (energética, transporte y telecomunicaciones) en el crecimiento de once municipios y siete ciudades de la frontera norte de México, a través de un análisis de panel. Los autores presentan evidencia de que la infraestructura social no posee efectos significativos y que la infraestructura productiva afecta positivamente al crecimiento económico, en especial, la infraestructura en energía, telecomunicaciones y transporte.

\section{ESPECIFICACIÓN DEL MODELO ECONOMÉTRICO}

En este apartado se define la metodología que se sigue para analizar los efectos del gasto público sobre el crecimiento de la producción de los 32 estados de México durante el período 2004-2018, controlando por la influencia de otras variables explicatorias relevantes. La especificación parte del siguiente modelo

11 Fuentes y Mendoza (2003) especifican una regresión de corte transversal y utilizan el método de estimación de mínimos cuadrados no lineales. Consideran como variables de control la infraestructura pública, representada por los parques industriales privados estatales, y el número de parques industriales en los estados como proxy del capital privado.

12 Ciudad de México, Guanajuato, Hidalgo, Estado de México, Morelos, Puebla, Querétaro y Tlaxcala.

13 El Índice de Infraestructura Productiva Social (IPS) está representado por el número de escuelas y el de Infraestructura Productiva Económica (IPE) agrupa tres subcategorías: Infraestructura energética, Infraestructura de transportes e Infraestructura de comunicaciones. 
general en el que las variables se agrupan en tres tipos: gasto público $\left(X_{1}\right)$, variables domésticas $\left(X_{2}\right)$ y variables del sector externo $\left(X_{3}\right)$,

$$
y=f\left(X_{1}, X_{2}, X_{3}\right)
$$

donde $y$ denota la tasa de crecimiento de la producción de los estados y $X_{1}$ contiene el crecimiento del gasto público corriente $(G C)$ y de inversión $(G I)^{14}$. Aunque se espera, de acuerdo con la literatura discutida previamente, que el gobierno pueda afectar positivamente al producto mediante el impulso de la demanda agregada, a través de las compras de bienes y servicios, los pagos de sueldos y salarios y el otorgamiento de subsidios, así como del crecimiento del potencial productivo, es posible que sus efectos distorsionantes lleven a la larga a un efecto adverso o nulo. Más aún, dado que los efectos de los componentes del gasto pueden ser diferentes se separan en gasto corriente y de inversión.

A su vez, $X_{2}$ contiene al índice de Herfindahl-Hirschman $(H H)$ que mide el nivel de concentración/diversificación de la estructura productiva de los sectores que integran el producto estatal ${ }^{15}$ y que puede afectar al crecimiento del producto de manera positiva o negativa. En este sentido, Chen (2019) establece que la teoría convencional indica que la especialización económica puede promover el crecimiento, mientras que la estabilidad económica está asociada con economías diversificadas. Esta relación conflictiva entre especialización y diversidad ha sido cuestionada, ya que los científicos han sugerido que la especialización y la diversidad pueden coexistir en una economía regional ${ }^{16}$. Por su parte, Kim (2019) establece que la diversificación aumenta cuando los países avanzan, mientras que los países en desarrollo pueden beneficiarse de la concentración estratégica ${ }^{17}$.

14 Véase el apéndice A para detalles sobre la construcción de la base de datos aquí utilizada.

15 El índice resulta del promedio que pondera el porcentaje al cuadrado de la participación de cada sector en el PIB total estatal. Varía originalmente entre 0 y 10 000, donde el primero corresponde a una situación de completa diversificación y el segundo de concentración en un solo sector. Para tener cifras comparables con las de las tasas de crecimiento del PIB, los resultados se dividieron por 1000 .

16 Los resultados empíricos de Chen (2019) confirman que el clúster y la diversidad contribuyen a la estabilidad económica y al crecimiento al mismo tiempo. Como tal, las regiones vecinas deben trabajar juntas para fortalecer la colaboración de diversas especializaciones y fomentar el desempeño económico.

17 El autor usa el índice de Herfindahl-Hirschman como medida de concentración de I+D, y encuentra que un nivel de concentración puede ser bueno para el crecimiento. Sin embargo, más allá de cierto punto, la concentración tiene un efecto negativo sobre el crecimiento (Kim, 2019). 
Por otro lado, $X_{3}$ recolecta indicadores de apertura de las economías estatales con el objetivo de medir su grado de inserción a la economía internacional. En ese sentido, $X_{3}=[B C, R R E M]$, donde la primera denota la tasa de crecimiento de bienes comerciables (sector básico) ${ }^{18}$ y la segunda a la razón de remesas recibidas en cada estado con respecto al producto correspondiente ${ }^{19}$. Dado el alto grado de correlación de la producción nacional y estatal con la demanda externa, principalmente de las exportaciones a Estados Unidos, se espera que la producción de bienes comerciables, principalmente de manufacturas, tuviera un efecto positivo sobre la producción estatal, como han documentado Mejía y Vergara (2017) y Mejía, Rendón, Vergara y Aroca (2018). Similarmente, se esperaría que los ingresos por remesas tuvieran un efecto positivo, especialmente en los estados en los que las remesas son una fuente importante de financiamiento del consumo y la construcción de las familias (Meyer \& Shera, 2017; Mendoza-Cota \& Torres-Preciado, 2019). No obstante, es posible que las remesas tengan una relación negativa con el producto en virtud de que son más importantes en estados menos relacionados con la dinámica productiva del resto del país y del exterior ${ }^{20}$ (Mejía et al., 2018; Aragonés, Salgado \& Ríos, 2008).

Adicionalmente, la Nueva Geografía Económica ha enfatizado el papel de las externalidades regionales según las cuales los países pueden beneficiarse de una mayor actividad económica en sus regiones geográficas cercanas, debido a que las empresas crean vínculos hacia adelante y hacia atrás (López-Bazo, Vaya \& Artis, 2004). Esto es, según el modelo centro-periferia, si existen algunos costos de transporte, la proximidad a las empresas que suministran productos intermedios reduce los costos y da lugar a vínculos de costos (o hacia adelante), en tanto que la presencia de empresas que utilizan bienes intermedios aumenta las ventas y las ganancias de los proveedores de bienes intermedios y crea vínculos de demanda

18 La producción del sector de bienes comerciables (o básicos) se asocia a la de los sectores de la agricultura, ganadería, silvicultura, pesca, caza, minería, manufactura, servicios de alojamiento y preparación de alimentos y bebidas.

19 La especificación original también incluyó la razón de inversión extranjera a PIB, pero no resultó significativa en especificación alguna, por lo que se decidió excluirla. Vale la pena mencionar que esta variable adolece del llamado problema del "domicilio fiscal" según el cual las inversiones se registran en el domicilio de la matriz (usualmente la Ciudad de México), aunque se llevan a cabo en estados diferentes.

20 Aragonés et al. (2008) sostienen que aunque las remesas permiten generar un mayor crecimiento y desarrollo económico en el país por medio del multiplicador del ingreso, este se ve anulado por la estrechez del mercado y el volumen de importaciones. Por su parte, Mendoza-Cota y Torres-Preciado (2019) establecen que en la etapa de apertura e integración económica de México se han generado montos elevados de remesas, pero no está claro el vínculo directo entre estos recursos financieros y la forma en que se han utilizado para estimular al crecimiento económico regional. 
(o hacia atrás). La interacción de estas fuerzas crea externalidades pecuniarias ${ }^{21}$, fomentando la aglomeración de la industria en una región de modo que, si estas fuerzas son lo suficientemente fuertes, la industria se concentrará en una sola región, pero en algún momento la industria se desborda en una serie de oleadas hacia otras regiones cercanas, y así sucesivamente, alcanzando eventualmente una masa crítica (Krugman \& Venables, 1995; Puga \& Venables, 1996).

Por tanto, para captar posible interacción espacial entre las tasas de crecimiento del producto de los diferentes estados (autocorrelación sustantiva) y/o entre los residuos de los modelos estimados (autocorrelación residual), se plantea la estimación de modelos espaciales para datos de panel, de acuerdo a la propuesta de Elhorst (2014). Para ello, siguiendo el enfoque "de lo general a lo particular", se parte del modelo espacial anidado general (GNS, por sus siglas en inglés) definido como:

$$
\begin{gathered}
Y_{t}=\rho W Y_{t}+\alpha \iota_{N}+X_{t} \beta+W X_{t} \theta+\mu+u_{t} \\
u_{t}=\lambda W u_{t}+\varepsilon_{t}
\end{gathered}
$$

donde $Y$ es el vector que contiene la tasa de crecimiento del PIB estatal como variable explicatoria, con $t=15$ períodos; $\iota_{N}$ es un vector de 1's, con $N=32$ estados, y $\alpha$ es el intercepto; $\mu=\left[\mu_{1} \mu_{2} \ldots \mu_{N}\right]$ es un conjunto de variables dicótomas que buscan capturar el efectos de variables omitidas propias de cada unidad considerada (excepto una para evitar multicolinealidad perfecta) e invariantes a través del tiempo, es decir, tratan de modelar la heterogeneidad espacial; $X=\left[X_{1} X_{2} X_{3}\right]$ es una matriz que contiene las variables explicatorias agrupadas en los tres tipos mencionados, con parámetros asociados contenidos en el vector $\beta$, en tanto que $u$ denota el vector de perturbaciones que sigue el proceso indicado en la expresión (3) y $\varepsilon$ sigue un proceso ruido blanco, es decir $\varepsilon \sim$ iid $N\left(0, \sigma^{2} I\right)$.

La ventaja principal de la especificación (2)-(3) es que anida formulaciones más sencillas que capturan diferentes efectos de interacción entre el crecimiento de la producción de los estados y el de sus vecinos. Definiendo $W$ como la matriz de pesos espaciales ${ }^{22}$, el primer término de (2) $W Y$ captura los efectos de interacción

21 Las externalidades que eventualmente llevan al surgimiento de un patrón centro-periferia son las externalidades pecuniarias, asociadas con vínculos de demanda o de oferta, más que derramas tecnológicas puras (Krugman, 1991). Las externalidades pecuniarias pueden conducir a las empresas a macroáreas que abarcan varias regiones, transfiriendo así las externalidades a nivel de empresa, al nivel regional agregado (López-Bazo et al., 2004).

$22 W$ es una matriz cuadrada de orden $(32 \times 32)$ que busca capturar la vecindad espacial, en este caso 
endógenos según los cuales el crecimiento de un estado depende también del de los estados vecinos, lo que resulta de la determinación simultánea del equilibrio en la vecindad espacial; $\rho$ es el parámetro que mide la también llamada dependencia espacial sustantiva entre cada estado y sus vecinos. A su vez, $W X$ representa los efectos de interacción exógenos, donde el comportamiento del crecimiento de cada estado mexicano también depende de las variables explicatorias de sus vecinos; el vector de parámetros $\theta$ mide estos efectos. Finalmente, la ecuación (3) representa los efectos de interacción entre los términos de error $(W u)$ que pueden resultar de la posible omisión de variables explicatorias o de la autocorrelación entre choques no observados; $\lambda$ mide estos efectos.

Entonces, siguiendo la metodología de "lo general a lo particular", se inicia estimando el modelo GNS definido en la formulación (2)-(3) y se evalúan diferentes especificaciones mediante la prueba de razón de verosimilitud $(L R)$. En ese sentido, se contrastan diferentes hipótesis para elegir entre el modelo general (no restringido) y el particular (restringido). Por ejemplo, si en el modelo GNS resulta que la hipótesis nula $\theta=0$ no se puede rechazar, se opta por el llamado modelo SAC, o si la evidencia no permite rechazar que $\lambda=0$ el modelo más apropiado sería el espacial de Durbin (SDM). Las pruebas de selección del modelo y las estimaciones correspondientes se presentan en la siguiente sección.

\section{RESULTADOS ECONOMÉTRICOS}

En este apartado se presentan las características generales de los datos y se discuten los resultados de los modelos espaciales de panel de datos estimados ${ }^{23}$. En ese sentido, en la figura 1 se presenta la distribución espacial de las tasas de crecimiento promedio de la producción entre 2004 y 2018. Campeche, estado altamente especializado en la producción de petróleo crudo, tiene el crecimiento más bajo del periodo (-4.4\%), como consecuencia del agotamiento paulatino de los yacimientos y la caída el precio internacional de ese producto (Banco de México [Banxico], 2018; Erquizio \& Ramírez, 2014). En el siguiente rango más bajo, de -4.4 a 1.9\%, destacan los estados del sur y los de la costa del océano Pacífico, que tradicionalmente han mostrado tasas reducidas como resultado de una serie de factores entre los que destacan su especialización en productos agropecuarios, limitada dotación

tipo reina, con entradas iguales a 1 si los estados comparten una frontera y 0 de otro modo, y estandarizada por renglón.

23 Es importante aclarar que con el objetivo de evitar estimaciones espurias, se ha optado por trabajar con transformaciones estacionarias de las series, tasas de crecimiento o razones con respecto al PIB. 
de infraestructura y bajos niveles educativos, entre otros (Banco Mundial [BM], 2003). A su vez, varios estados localizados en la frontera norte, centro-norte y centro del país muestran tasas de crecimiento elevadas (2.6-3.6\%) y se caracterizan por el haber desarrollado la producción industrial en el transcurso de las últimas décadas, así como por aparentemente beneficiar a sus estados vecinos (Carbajal et al., 2016). Llama la atención que estos dos grupos de estados se concentren en regiones específicas y que compartan fronteras, patrón que contrasta con el de los estados que tienen las tasas más bajas (ya mencionado) y las más altas (3.6-5.8\%), que se hallan dispersos a lo largo del territorio nacional.

Figura 1. Tasas de crecimiento promedio producto interno bruto. 2004-2018

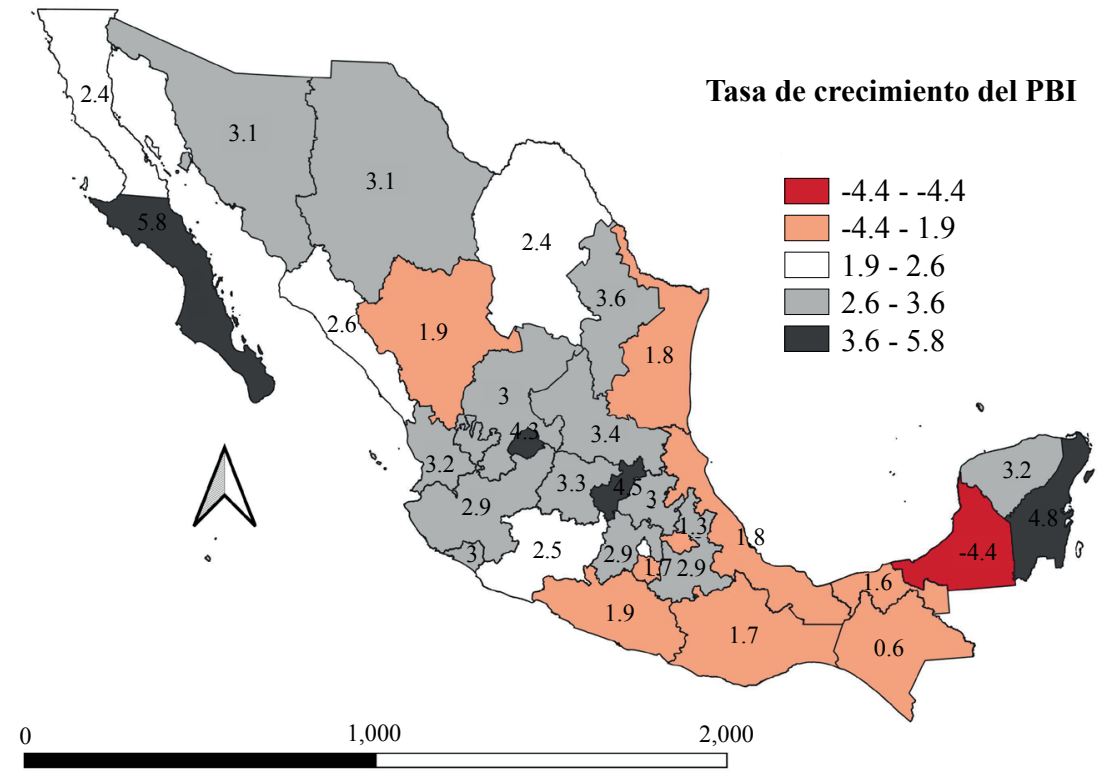

Fuente: elaboración propia.

Se obtuvieron las medias y las desviaciones estándar de las variables de estudio $^{24}$. De acuerdo a las últimas, todas las variables, sobre todo el gasto público corriente y de inversión, presentan un comportamiento muy diferenciado entre los estados del país. Por ejemplo, las razones remesas/PIB superiores al promedio

24 Estos datos están a disposición. 
nacional se presentan en Guerrero (15.1\%) y Michoacán (10.3\%), en tanto que el crecimiento de la producción de bienes comerciables ha sido negativa en la Ciudad de México $(-0.9 \%)^{25}$ y Campeche (-5.1\%). Por su lado, dado que sus valores varían entre 0 y $10,{ }^{26}$ el índice de Herfindahl-Hirschman sugiere una significativa diversificación de la estructura productiva de los estados, a excepción de Campeche (6.9) y Tabasco (2.9), cuya producción se concentra altamente en el sector petrolero.

Con respecto al gasto público, las cifras reflejan tasas de crecimiento muy heterogéneas, con una media nacional para el gasto corriente y el gasto de inversión iguales 4.3 y $13.5 \%$ y desviaciones estándar de 17.6 y $62.2 \%$, respectivamente, en tanto que sus participaciones en el PIB igualan 2.6 y $0.6 \%$, respectivamente. Sobresalen los estados de Tlaxcala e Hidalgo con las tasas de crecimiento promedio más bajas $(0.9$ y -4.7\%); Zacatecas y Nuevo León con la dispersión más elevada (64.9 y $157.5 \%$ ), y Aguascalientes y Tabasco con las menores participaciones en el PIB (0.9 y $0.1 \%$ ) y Michoacán y Oaxaca con las mayores ( 7.3 y $2.1 \%$ ). Esta situación refleja, por un lado, el peso del gasto de inversión como resultado de la aplicación de la corriente neoliberal, cuyos fundamentos económicos no modifican, en esencia, la asignación presupuestaria en obras de infraestructura, pero sí el financiamiento con capital privado, y que han generado un cambio en la función del sector público, ahora centrado en la estabilidad macroeconómica. Por otro lado, se percibe la existencia de desigualdades crecientes en la dotación de recursos públicos en el territorio mexicano.

Los efectos del gasto público y de otras variables de control sobre la producción se analizan en el marco del modelo especificado en las expresiones (2)(3); los resultados se despliegan en la tabla 1, donde aparecen los coeficientes estimados y las pruebas de las distintas especificaciones. Como se mencionó en la metodología, para elegir la especificación más adecuada del modelo se aplican pruebas de razón de verosimilitud $(L R)$ convencionales que contrastan los modelos "sin restricciones" y "con restricciones". Además, se verifica que los parámetros relevantes $(\theta, \beta, \rho, \lambda)$ sean significativos estadísticamente. Los modelos se estiman por el método de máxima verosimilitud e incorporan efectos espaciales fijos ${ }^{27}$.

25 La Gran Recesión parece haber afectado más a la Ciudad de México como resultado de su especialización en subsectores poco competitivos y dinámicos. No obstante, durante las últimas décadas se ha ido desconcentrando su actividad industrial hacia los estados vecinos, consolidándose, como el polo dominante de servicios (Rendón, Mejía \& Díaz, 2021).

26 Recuérdese que $H H$ se ha dividido por 1000.

27 La prueba de Hausman del modelo de datos agrupados sugiere rechazar la hipótesis nula de igualdad de los parámetros del modelo (valor $p=0.006$ ), lo que implica incorporar efectos fijos. Se estimaron también los modelos con efectos fijos de tiempo y espaciales y de tiempo conjuntamente, pero en todos ellos se perdía la significancia estadística de los parámetros de autocorrelación espacial. 
Entonces, se procedió a estimar el modelo espacial anidado general (GNS) y, con base en la prueba $L R$, se concluye que la hipótesis nula $H_{0}: \theta=0$ no se rechaza, resultado que se puede explicar porque la mayoría de las variables explicativas rezagadas espacialmente no son estadísticamente significativas. En tal caso, se debería optar por el modelo SAC, aunque solamente el coeficiente de autocorrelación sustantiva $\rho$ es significativo, pero no el de autocorrelación residual $\lambda$. Efectivamente, las pruebas para contrastar los modelos $\operatorname{SAR}\left(H_{0}: \lambda=0\right)$ y $\operatorname{SEM}\left(H_{0}: \rho=0\right)$ con el SAC (que los anida) sugieren no rechazar la primera de estas hipótesis y rechazar la segunda, por lo que el modelo SAR sería una elección apropiada.

Alternativamente, se puede evaluar si los modelos espacial de Durbin (SDM) y de error espacial de Durbin (SDEM) están anidados en el modelo GNS evaluando las hipótesis nulas $H_{0}: \lambda=0$ y $H_{0}: \rho=0$, respectivamente. Los estadísticos correspondientes sugieren no rechazar la primera y hacerlo con la segunda, por lo que se optaría por el modelo SDM y por el GNS, aun cuando $\lambda$ no es significativa estadísticamente en el último, pero sí en el SDEM. De manera similar se contrastan los modelo SAR y SEM contra los SDM y SDEM, respectivamente, evaluando la hipótesis nula $H_{0}: \theta=0$ en cada caso. Los estadísticos $L R$ implican el rechazo de la hipótesis nula en el modelo SDM y el no rechazo en el SDEM. No obstante, como se aprecia en la tabla 1, los coeficientes de las variables rezagadas espacialmente no son estadísticamente significativas en el modelo SDM. Por lo tanto, la estrategia "de lo general a lo particular" conduce a las versiones más restringidas, los modelos SAR y SEM ${ }^{28}$.

Tabla 1. Estimaciones de modelos para datos de panel con efectos fijos espaciales

\begin{tabular}{lccccccc}
\hline \multirow{2}{*}{$\begin{array}{c}\text { Variables } \\
\text { explicatorias }\end{array}$} & GNS & SAC & SDM & SDEM & SAR & SEM & OLS \\
\cline { 2 - 8 } & $\begin{array}{ccccccc}-0.262 \\
\text { RREM }\end{array}$ & $\begin{array}{c}-0.148 \\
(0.172)\end{array}$ & $\begin{array}{c}-0.159 \\
(0.135)\end{array}$ & $\begin{array}{c}-0.053) \\
(0.012)\end{array}$ & $\begin{array}{c}-0.179 \\
0.091)\end{array}$ & $\begin{array}{c}-0.320 \\
(0.013)\end{array}$ & $\begin{array}{c}-0.091 \\
(0.002)\end{array}$ \\
\hline \multirow{2}{*}{$B C$} & 0.407 & 0.409 & 0.416 & 0.430 & 0.415 & 0.430 & 0.489 \\
& $(0.000)$ & $(0.000)$ & $(0.000)$ & $(0.000)$ & $(0.000)$ & $(0.000)$ & $(0.000)$ \\
\hline
\end{tabular}

28 Es interesante observar que el criterio de información de Akaike (AIC) se minimiza con el modelo SDM y que su segundo valor más bajo corresponde al SAR. Similarmente, el criterio de información bayesiano de Schwarz (BIC) alcanza su mínimo con el modelo SAR. Más todavía, las pruebas $L M$ (LM-Lag y LM-Error), obtenidas a partir de la estimación del modelo de datos agrupados, sugieren también la estimación de cualquiera de estos dos modelos, de acuerdo a la metodología de Anselin (1996). En el mismo sentido, el índice de Moran sugiere la existencia de dependencia espacial global. 
EFECTOS DEL GASTO PÚBLICO SOBRE LA PRODUCCIÓN DE LOS ESTADOS DE MÉXICO, 2004-2018: ...

\begin{tabular}{|c|c|c|c|c|c|c|c|}
\hline$H H$ & $\begin{array}{c}1.944 \\
(0.013)\end{array}$ & $\begin{array}{c}1.893 \\
(0.016)\end{array}$ & $\begin{array}{l}1.755 \\
(0.021)\end{array}$ & $\begin{array}{c}1.105 \\
(0.123)\end{array}$ & $\begin{array}{c}1.772 \\
(0.019)\end{array}$ & $\begin{array}{c}1.125 \\
(0.114)\end{array}$ & $\begin{array}{l}-0.626 \\
(0.000)\end{array}$ \\
\hline$G C$ & $\begin{array}{c}0.001 \\
(0.790)\end{array}$ & $\begin{array}{c}0.001 \\
(0.746)\end{array}$ & $\begin{array}{c}0.002 \\
(0.665)\end{array}$ & $\begin{array}{c}0.001 \\
(0.690)\end{array}$ & $\begin{array}{c}0.001 \\
(0.725)\end{array}$ & $\begin{array}{c}0.001 \\
(0.709)\end{array}$ & $\begin{array}{l}-0.002 \\
(0.642)\end{array}$ \\
\hline$G I$ & $\begin{array}{l}-0.000 \\
(0.962)\end{array}$ & $\begin{array}{l}-0.000 \\
(0.955)\end{array}$ & $\begin{array}{r}-0.000 \\
(0.865)\end{array}$ & $\begin{array}{l}-0.000 \\
(0.840)\end{array}$ & $\begin{array}{c}-0.000 \\
(0.938)\end{array}$ & $\begin{array}{l}-0.000 \\
(0.823)\end{array}$ & $\begin{array}{l}-0.001 \\
(0.359)\end{array}$ \\
\hline WRREM & $\begin{array}{c}0.074 \\
(0.154)\end{array}$ & & $\begin{array}{c}0.045 \\
(0.381)\end{array}$ & $\begin{array}{c}0.031 \\
(0.561)\end{array}$ & & & \\
\hline$W B C$ & $\begin{array}{c}0.035 \\
(0.236)\end{array}$ & & $\begin{array}{c}0.033 \\
(0.254)\end{array}$ & $\begin{array}{c}0.053 \\
(0.073)\end{array}$ & & & \\
\hline WHH & $\begin{array}{c}0.168 \\
(0.374)\end{array}$ & & $\begin{array}{c}0.126 \\
(0.488)\end{array}$ & $\begin{array}{c}-0.012 \\
(0.943)\end{array}$ & & & \\
\hline$W G C$ & $\begin{array}{l}-0.010 \\
(0.312)\end{array}$ & & $\begin{array}{l}-0.016 \\
(0.132)\end{array}$ & $\begin{array}{c}-0.005 \\
(0.508)\end{array}$ & & & \\
\hline$W G I$ & $\begin{array}{c}0.003 \\
(0.244)\end{array}$ & & $\begin{array}{c}0.002 \\
(0.265)\end{array}$ & $\begin{array}{c}0.001 \\
(0.480)\end{array}$ & & & \\
\hline
\end{tabular}

Constante

(0.000)

\begin{tabular}{|c|c|c|c|c|c|}
\hline$\rho$ & $\begin{array}{c}0.334 \\
(0.000)\end{array}$ & $\begin{array}{c}0.332 \\
(0.000)\end{array}$ & $\begin{array}{c}0.287 \\
(0.000)\end{array}$ & & \\
\hline$\lambda$ & $\begin{array}{l}-0.081 \\
(0.450)\end{array}$ & $\begin{array}{l}-0.066 \\
(0.525)\end{array}$ & & $\begin{array}{c}0.407 \\
(0.000)\end{array}$ & $\begin{array}{c}0.398 \\
(0.000)\end{array}$ \\
\hline
\end{tabular}

$\begin{array}{lllllll}\log L & -982.718 & -985.871 & -964.547 & -993.947 & -986.072 & -996.642\end{array}$

$\begin{array}{llllllll}\text { AIC } & 1991.437 & 1987.743 & 1973.094 & 2011.894 & 1986.145 & 2007.285 & 2092.044\end{array}$

$\begin{array}{llllllll}\text { BIC } & 2045.696 & 2021.133 & 2064.918 & 2061.981 & 2015.362 & 2036.502 & 2117.087\end{array}$

\begin{tabular}{|c|c|c|c|c|c|c|c|}
\hline $\mathrm{R}^{2}$ & 0.225 & 0.225 & 0.169 & 0.3468 & 0.2450 & 0.3425 & 0.682 \\
\hline $\begin{array}{l}\text { LR test } \\
\left(\mathrm{H}_{0}: \theta=0\right)\end{array}$ & $\begin{array}{c}6.260 \\
(0.282)\end{array}$ & $\begin{array}{c}6.311 \\
(0.277)\end{array}$ & & & $\begin{array}{l}43.051 \\
(0.000)\end{array}$ & $\begin{array}{c}5.390 \\
(0.370)\end{array}$ & \\
\hline
\end{tabular}




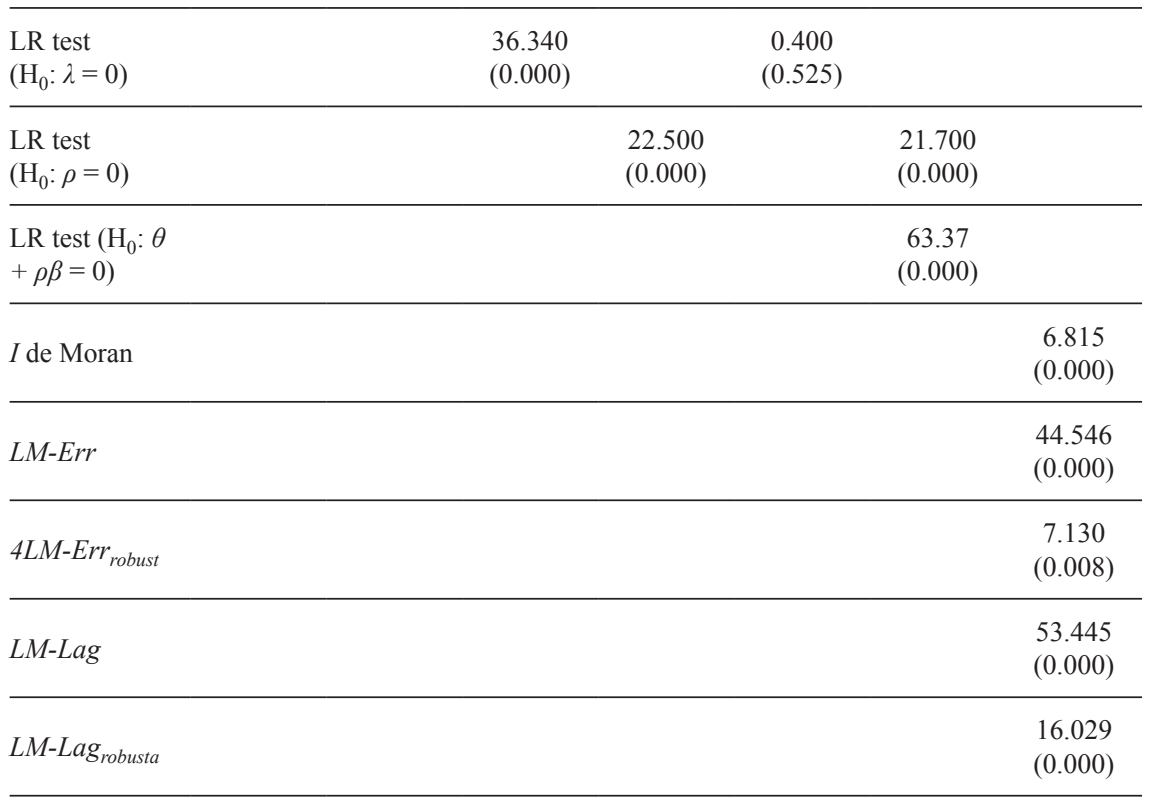

Fuente: elaboración propia.

En general, llama la atención la robustez (robustness) de los coeficientes estimados para las diferentes especificaciones, tanto en términos de signos como de magnitud, como se ve en la tabla 1. Específicamente, acorde con otros estudios, el sector de bienes comerciables tiene un efecto positivo sobre la producción, lo que refleja el importante papel de las manufacturas como impulsor del crecimiento en los estados más vinculados al sector exportador (frontera norte, centro-norte y centro) ${ }^{29}$ (Mejía \& Vergara, 2017), pero también de otros sectores relevantes en estados específicos, como el turismo (Quintana Roo, Yucatán y Baja California Sur) o el sector agroexportador (Sinaloa, Sonora, Michoacán y Jalisco). Por el contrario, los estados con menores tasas de crecimiento son también los menos industrializados y con una vinculación mínima al exterior.

La razón de remesas a PIB, a su vez, tiene un coeficiente con signo negativo, acorde a la hipótesis de suavizamiento ${ }^{30}$ (Sayan, 2006; Frankel, 2009) apoyada

29 De hecho, varios estudios han argumentado que la manufactura sigue siendo el sector motriz de la economía, acorde con las hipótesis de Kaldor, entre estos el de Loría, Moreno-Brid, Salas \& Sánchez-Juárez (2019).

30 Según la "hipótesis de suavizamiento", las remesas pueden ser contracíclicas en el país de origen del 
también por los hallazgos de Mejía et al. (2018) para los estados de México. Esencialmente, este resultado sugiere que los estados que más remesas reciben como porcentaje de su producto, es decir, que están más integrados al mercado laboral de los Estados Unidos, principalmente, son también las que tienen menores ritmos de crecimiento (Mendoza-Cota \& Calderón, 2006; Mendoza \& Valdivia, 2016).

Los coeficientes estimados de $H H$ sugieren que, dado que se parte de un alto grado de diversificación de la estructura productiva estatal, un aumento de la concentración de la producción sectorial genera un mayor ritmo de crecimiento productivo de los estados, y viceversa, lo que podría explicarse por las ganancias resultantes de la especialización en actividades productivas particulares. De hecho, los estados que han crecido a mayores ritmos se han ido especializando paulatinamente en algunos sectores dinámicos clave como el de equipo de transporte (Guanajuato, Coahuila, Puebla), aeronáutica y agroalimentaria (Querétaro), automotriz (San Luis Potosí, Aguascalientes, Sonora), Manufactura-Turismo (Baja California Sur y Quintana Roo), agroalimentaria (Morelos) y manufactura en general (Nuevo León) (Rendón, Mejía \& Vergara, 2019; Erquizio \& Ramírez, 2014).

Por último, resulta por demás interesante que las tasas de crecimiento del gasto público, corriente y de inversión, no tengan efecto alguno sobre el crecimiento de la producción, resultado plausible, aunque no esperado ante la evidencia reportada por diferentes estudios del caso de los estados mexicanos (Arwiphawee \& Sánchez, 2015; Fuentes, 2003). No obstante, otros estudios, como Mejía \& Sánchez (2019), sugieren que la relación entre gasto público y producción es débil, inestable y limitada a pocos componentes del primero, en tanto que Fuentes y Mendoza (2003) sostienen que esa relación se desvanece entre 1985 y 1998. En ese sentido, nuestros hallazgos podrían explicarse por diferentes factores entre los que destaca la alta volatilidad del crecimiento del gasto (sugerido por su alta desviación estándar), que podría ser un indicador del alto grado de discrecionalidad en su manejo (Reyes \& Mejía, 2016), así como por el limitado alcance del quehacer público resultante de su pequeña magnitud en comparación con el PIB estatal ${ }^{31}$.

Más aún, existen otros factores que podrían contribuir a explicar el nulo efecto productivo del gasto público en los estados de México, entre los que destaca

trabajador (el receptor de remesas), pero procíclicas en el país de acogida del migrante (el remitente). Por tanto, pueden actuar como factor estabilizador en el país receptor.

31 También se podría conjeturar que el efecto desplazamiento del gasto público sobre la inversión privada podría ser una causa relevante. No obstante, la ausencia de información estadística sobre inversión y tasas de interés específicas hacen difícil evaluar esta hipótesis a nivel estatal en México. 
el uso ineficiente de los recursos. De hecho, los estudios internacionales sobre competitividad de los países han documentado importantes volúmenes de desviación de recursos públicos a nivel nacional favorecida por los altos niveles de corrupción de diferentes gobiernos (WEF, varios años) ${ }^{32}$, así como por la asignación del gasto público estatal a actividades no productivas (Lambarri, 2021) (33. $^{3}$.

Como se indicó más arriba, el modelo más apropiado para explicar el crecimiento del producto estatal es el SAR. Las propiedades de los coeficientes de las variables explicatorias son similares a los patrones descritos, con RREM, BC y $H H$ significativas estadísticamente ${ }^{34}$ y GC y GI no significativas. Solo falta agregar que la autocorrelación sustantiva es estadísticamente significativa al $1 \%$ de significancia y positiva, con un coeficiente $\rho=0.30$, lo que significa que las variaciones del producto de las entidades federativas son explicadas en esa proporción por el crecimiento de las entidades vecinas. Es decir, este resultado significa que existen dinámicas virtuosas de retroalimentación y derrame entre estados vecinos.

Entonces, la existencia de autocorrelación espacial estadísticamente significativa da pie a la posibilidad de descomponer los resultados del modelo SAR para identificar tanto los efectos de las variables explicatorias sobre el crecimiento del producto de la misma unidad espacial (efectos directos) como los provenientes de las mismas variables en las unidades vecinas derivados de los efectos de retroalimentación (efectos indirectos). Las estimaciones, mostradas en la tabla 2, sugieren que el crecimiento del producto depende principalmente de los efectos de las variables de control (RREM, HH y $B C$ ) de la misma unidad espacial, con alrededor del $71.3 \%$, y en menor medida de lo que acontece en la vecindad, con un porcentaje que ronda el $28.5 \%$. Estos porcentajes se reflejan también en la magnitud de los coeficientes correspondientes. Por ejemplo, el crecimiento de $1 \%$ de la producción de bienes comerciables de un estado se ve reflejado en un aumento de $0.4 \%$ del producto del mismo estado, pero un alza similar de la producción del mismo sector

32 Según el Foro Económico Mundial (WEF en inglés), las instituciones públicas de México han tenido uno de los desempeños más pobres en comparación con la calificación de otros países. Los resultados de 2015 muestran un deterioro significativo que refleja el clima de desconfianza e incertidumbre en las instituciones mexicanas, entre los rubros con las peores posiciones están el crimen organizado (140), costos empresariales de la delincuencia y la violencia (135), confianza en los servicios de policía (128) y desvío de fondos públicos (119).

33 Lambarri (2021) argumenta que en 2019 la suma en el gasto en materia de salud, educación, justicia y seguridad representa más del $60 \%$ del total de los recursos ejercidos durante ese año en 21 entidades federativas. En las otras once entidades, el bienestar social en realidad no es una prioridad.

La primera al 10\% y las dos siguientes al 5\%. 
en la vecindad provoca un aumento menor en el estado en cuestión, $0.2 \%$. El resto de los efectos directos e indirectos estimados se interpretan de manera similar.

El peso de los efectos directos e indirectos cambia en el caso del gasto corriente $(G C)$ y de inversión $(G I)$ de los gobiernos de los estados: Los resultados indican que la mayor proporción correspondería a los efectos directos en ambos casos, pero de cualquier modo las estimaciones siguen siendo no significativas estadísticamente.

En cuanto a los impactos indirectos, identificamos efectos de arrastre o desbordamiento espaciales entre los estados, dado que sus valores estimados son significativamente distintos de cero. Este resultado pone de manifiesto que los cambios iniciales en estas variables, de todas las entidades respecto a un estado $i$ aumenta el nivel de la producción, dada la interdependencia entre las entidades mexicanas. Esto no sucede con las variables del gasto las cuales no son estadísticamente significativas, y cuyo valor del coeficiente es cero.

Tabla 2. Efectos directos e indirectos sobre el PIB. SAR con efectos fijos espaciales

\begin{tabular}{|c|c|c|c|}
\hline & Coeficiente & $\begin{array}{c}\text { Porcentaje } \\
\text { en el efecto total }\end{array}$ & $\begin{array}{l}\text { Porcentaje de } \\
\text { retroalimentación }\end{array}$ \\
\hline \multicolumn{4}{|c|}{ Efectos directos } \\
\hline RREM & $\begin{array}{l}-0.187 \\
(0.080)\end{array}$ & 71.374 & -0.800 \\
\hline $\mathrm{BC}$ & $\begin{array}{c}0.427 \\
(0.000)\end{array}$ & 71.404 & 1.201 \\
\hline $\mathrm{HH}$ & $\begin{array}{l}1.841 \\
(0.012)\end{array}$ & 71.328 & 6.925 \\
\hline GC & $\begin{array}{l}0.001 \\
(0.745)\end{array}$ & 99.856 & -0.010 \\
\hline GI & $\begin{array}{l}0.000 \\
(0.970) \\
\end{array}$ & 97.139 & 0.003 \\
\hline \multicolumn{4}{|c|}{ Efectos indirectos } \\
\hline RREM & $\begin{array}{l}-0.074 \\
(0.092)\end{array}$ & 28.244 & \\
\hline $\mathrm{BC}$ & $\begin{array}{c}0.171 \\
(0.000)\end{array}$ & 28.595 & \\
\hline
\end{tabular}




\begin{tabular}{|c|c|c|}
\hline $\mathrm{HH}$ & $\begin{array}{c}0.739 \\
(0.023)\end{array}$ & 28.632 \\
\hline $\mathrm{GC}$ & $\begin{array}{l}0.000 \\
(0.732)\end{array}$ & 0.146 \\
\hline GI & $\begin{array}{c}-0.000 \\
(0.983)\end{array}$ & 2.870 \\
\hline \multicolumn{3}{|c|}{ Efecto Total } \\
\hline RREM & $\begin{array}{c}-0.262 \\
(0.080)\end{array}$ & \\
\hline $\mathrm{BC}$ & $\begin{array}{c}0.598 \\
(0.000)\end{array}$ & \\
\hline $\mathrm{HH}$ & $\begin{array}{l}2.581 \\
(0.013)\end{array}$ & \\
\hline $\mathrm{GC}$ & $\begin{array}{c}0.002 \\
(0.741)\end{array}$ & \\
\hline GI & $\begin{array}{l}-0.000 \\
(0.974)\end{array}$ & \\
\hline
\end{tabular}

Fuente: elaboración propia.

Los efectos totales son mayores a los coeficientes estimados directamente en el modelo SAR, esto como consecuencia de los efectos de retroalimentación ${ }^{35}$, que se refieren al proceso en el que un efecto originado en un estado en particular pasa a los estados vecinos y regresa a la entidad federativa donde inició. Estos efectos de retroalimentación se deben en parte al coeficiente de variable dependiente espacialmente rezagada, y en parte al coeficiente del valor espacialmente rezagado de la variable explicativa en sí.

En ese sentido, se observa que los efectos de retroalimentación resultan ser pequeños para las remesas, de $-0.8 \%$ del efecto directo, del mismo modo que el efecto de retroalimentación de la variable de los bienes comerciables que asciende a 1.2\% del efecto directo. En cambio, existe una retroalimentación importante entre las entidades federativas con el índice de Herfindahl-Hirschman de 6.9\%. Lo que implica que si las remesas, los bienes comerciables y la especialización contribuyen al aumento de la producción de un estado en particular, también lo hacen en sus estados vecinos.

35 El cálculo es la diferencia entre el efecto directo y el coeficiente $\beta \beta$ respectivo. 


\section{CONCLUSIONES}

En este documento se han analizado los efectos del crecimiento del gasto público, distinguiendo entre gasto corriente y de inversión, sobre el crecimiento de la producción en los estados de México durante el período 2004-2018. Para ello, se han estimado modelos espaciales para datos de panel, seleccionados de acuerdo al enfoque "de lo general a lo particular", que toman en cuenta el papel de otras variables relevantes.

Los resultados sugieren la existencia de autocorrelación espacial sustantiva (modelo SAR) que sugiere la presencia de interacciones entre el crecimiento de la producción de estados vecinos. Cuando se toman en cuenta los efectos de retroalimentación, resulta que el crecimiento de la producción estatal depende principalmente de los efectos de las variables explicatorias de la misma unidad espacial (efectos directos), con alrededor de 71.3\%, y en menor medida de lo que acontece en la vecindad (efectos indirectos), con un porcentaje que ronda el $28.5 \%$. Es decir, este resultado significa que existen dinámicas virtuosas de retroalimentación y derrame entre estados vecinos que pueden explicarse por la existencia de economías externas, como sugiere la nueva geografía económica.

Las propiedades de los coeficientes y de los efectos directos, indirectos y totales de las variables explicatorias sugieren que el sector de bienes comerciables tiene un efecto positivo sobre la producción, lo que refleja el importante papel de las manufacturas, el turismo y el sector agroexportador como medios de inserción de la economía internacional de los estados especializados en estas actividades. Por el contrario, los estados con menores tasas de crecimiento son también los de menor vinculación con el exterior. En un sentido complementario, el índice HerfindahlHirschman presenta también efectos positivos, lo que sugiere que, partiendo de una base diversificada, un aumento de la concentración de la producción sectorial genera un mayor ritmo de crecimiento productivo de los estados debido a una mayor especialización productiva, lo que podría explicarse por la especialización en algunos sectores dinámicos clave, como los mencionados. Por su parte, la razón de remesas a PIB se asocia negativamente con el desempeño productivo, lo que confirma que los estados que proporcionalmente reciben más remesas son también las que tienen menores ritmos de crecimiento, y viceversa.

Finalmente, se encontró que las tasas de crecimiento del gasto público, corriente y de inversión no tienen efecto alguno sobre el crecimiento de la producción estatal, evidencia que se suma a la diversidad de resultados previamente 
reportados en la literatura nacional e internacional. Estos hallazgos marcan la pauta para ulteriores investigaciones que analicen explícitamente el papel de factores que aquí se han mencionado más como conjeturas que como explicaciones. Entre ellos destacan la composición del gasto a niveles más específicos, como la proporción destinada a sueldos y salarios, así como a programas sociales; la alta volatilidad de su crecimiento, que podría reflejar un alto grado de discrecionalidad en su manejo; su limitado alcance, resultante de su pequeña magnitud en comparación con el PIB a nivel estatal, y la desviación y el uso ineficiente de los recursos públicos, entre otros. 


\section{APÉNDICE}

\section{Cuadro A.1. Descripción de las variables}

\begin{tabular}{|c|c|c|c|c|c|}
\hline Variable & Descripción & Fuente & $\begin{array}{l}\text { Unidad de } \\
\text { medida }\end{array}$ & $\begin{array}{c}\text { Constantes/ } \\
\text { nominales }\end{array}$ & $\begin{array}{c}\text { Cálculo de la variable en } \\
\text { el modelo }\end{array}$ \\
\hline $\mathrm{GC}$ & $\begin{array}{c}\text { Variable proxy } \\
\text { del gasto } \\
\text { corriente }^{1}\end{array}$ & $\begin{array}{c}\text { INEGI. Estadísticas } \\
\text { de finanzas públicas } \\
\text { estatales y municipales. } \\
\text { https://www.inegi.org. } \\
\text { mx/programas/finanzas/ } \\
\text { default.html\#Datos_- } \\
\text { abiertos }\end{array}$ & $\begin{array}{l}\text { Cifras en } \\
\text { pesos }\end{array}$ & $\begin{array}{l}\text { Constantes } \\
\text { Base } 2013\end{array}$ & Tasas de crecimiento \\
\hline $\mathrm{HH}$ & $\begin{array}{l}\text { Índice de } \\
\text { Herfindahl- } \\
\text { Hirschman }\end{array}$ & $\begin{array}{c}\text { INEGI. Sistema de } \\
\text { Cuentas Nacionales de } \\
\text { México. } \\
\text { Producto Interno Bruto } \\
\text { por Entidad Federativa. }\end{array}$ & $\begin{array}{l}\text { Cifras en } \\
\text { pesos }\end{array}$ & $\begin{array}{l}\text { Constantes } \\
\text { Base } 2013\end{array}$ & $\begin{array}{l}\text { Se obtiene al cuantificar el } \\
\text { cuadrado de los porcenta- } \\
\text { jes de participación de cada } \\
\text { uno de los sectores que } \\
\text { conforman la economía. } \\
\text { Se reporta en escala entre } \\
0 \text { y } 10000 \text {, entre más con- } \\
\text { centrado esté un sector más } \\
\text { cercano a } 1 \text { se encuentra. } \\
\text { El índice se calcula con la } \\
\text { fórmula: } \\
I H H=\sum_{j=1}^{n}\left(\frac{x_{i j}}{X T_{i}}\right)^{2} \\
\text { Donde: } \\
x i j \text { indica la participación } \\
\text { de cada sector en el total } \\
\text { de la producción }(\mathrm{XT}) \text {. }\end{array}$ \\
\hline $\mathrm{BC}$ & $\begin{array}{l}\text { Sector básico } \\
\text { comerciable }^{2}\end{array}$ & $\begin{array}{c}\text { INEGI. Sistema de } \\
\text { Cuentas Nacionales de } \\
\text { México. }\end{array}$ & $\begin{array}{l}\text { Cifras en } \\
\text { pesos }\end{array}$ & $\begin{array}{l}\text { Constantes } \\
\text { Base } 2013\end{array}$ & Tasas de crecimiento \\
\hline RREM & Remesas & $\begin{array}{l}\text { INEGI. Banco de } \\
\text { Información Económica } \\
\text { (BIE). }\end{array}$ & $\begin{array}{l}\text { Cifras en } \\
\text { pesos }\end{array}$ & $\begin{array}{l}\text { Constantes } \\
\text { Base } 2013\end{array}$ & $\begin{array}{c}\text { Razón de remesas entre } \\
\text { PIB estatal }\end{array}$ \\
\hline
\end{tabular}

${ }^{1}$ Se suman los rubros clasificados por objeto del gasto: 1000 servicios personales, 2000 materiales y suministros y 3000 servicios generales.

${ }^{2}$ Incluye agricultura, ganadería, silvicultura, pesca, caza, minería, manufactura, servicios de alojamiento temporal y preparación de alimentos y bebidas.

Fuente: elaboración propia. 


\section{REFERENCIAS}

Anselin, L., Bera, A., Florax, R., \& Yoon, M. (1996). Simple Diagnostic Tests for Spatial Dependence. Regional Science and Urban Economics, 26(1), 77-104. doi: 10.1016/0166-0462(95)02111-6

Aragonés, A. M., Salgado, U., \& Ríos, E. (2008). ¿A quién benefician las remesas? Economía UNAM, 5(14), 37-55. Recuperado de http://www.scielo.org.mx/scielo.php?script=sci_arttext\&pid=S1665-952X2008000200002\&lng=es\&tlng=es Arslanalp, S., Bornhorst, F., \& Gupta, S. (2011). Inversión y crecimiento. Finanzas y Desarrollo, 48(1), 34-37. Recuperado de https://www.imf.org/external/ pubs/ft/fandd/spa/2011/03/pdf/Arslanalp.pdf

Arwiphawee, S., \& Sánchez, I. (2015). Fiscal policies and subnational economic growth in Mexico. International Journal of Economics and Financial Issues, 5(1), 11-22. Recuperado de https://www.econjournals.com/index. php/ijefi/article/view/962

Bagdigen, M., \& Cetintas, H. (2004). Causality between public expenditure and economic growth: The Turkish case. Journal of Economic and Social Research, 6(1), 53-72. Recuperado de https://mpra.ub.uni-muenchen.de/ $\mathrm{id} /$ eprint $/ 8576$

Bajo, O., \& Díaz, C. (2003). Política fiscal y crecimiento: nuevos resultados para las regiones españolas, 1967-1995. Investigaciones Regionales, 3, 99-111. Recuperado de: https://ebuah.uah.es/dspace/handle/10017/32349

Banco de México (2020). Reporte sobre las Economías Regionales. Recuperado de https://www.banxico.org.mx/publicaciones-y-prensa/reportessobre-las-economias-regionales/\%7B8427BCB2-D8F2-C28A-8DD4EB8DD9770681\%7D.pdf

Banco Mundial (2003). Estrategia de desarrollo de los estados del sur de México. Volumen 1: Resumen del informe. Recuperado de http://documents1.worldbank.org/curated/en/724461468757235721/pdf/311160SPANISH01outhSta tes0Synthesis.pdf

Barajas, H.A \& Gutiérrez, L. (2012). La importancia de la infraestructura física en el crecimiento económico de los municipios de la frontera norte. Estudios fronterizos, 13(25), 57-88. doi: 10.21670/ref.2012.25.a03

Barro, R. (1990). Government Spending in a Simple Model of Endogenous Growth. Journal of Political Economics, 98(5), 103-125. doi: 10.1086/261726

Barro, R. J., \& Sala-i-Martin, X. (1992). Public finance in models of economic growth, The Review of Economic Studies, 59(4), 645-661. doi: $10.2307 / 2297991$

Barro, R. J., \& Sala-i-Martin, X. (1995). Economic Growth. Boston: McGraw Hill. doi: 10.1016/S0165-1889(97)00007-9 
Carbajal, Y., Almonte, L., \& Mejía, P. (2016). La manufactura y la industria automotriz en cuatro regiones de México. Un análisis de su dinámica de crecimiento, 1980-2014. Economía: teoría y práctica, (45), 39-66. Recuperado de http://www.scielo.org.mx/scielo.php?script=sci_arttext\&pid=S0188$33802016000200039 \& \operatorname{lng}=$ es\&tlng=es

Chen, J. (2019). The Impact of Cluster Diversity on Economic Performance in U.S. Metropolitan Statistical Areas. Economic Development Quarterly, 34(1), 46-63. doi:10.1177/0891242419892338

Diaz, M. A., Mejía, P., Reyes, M. R., \& Desiderio, A. (2018). Efectos del gasto público en el PIB en los estados de México, 1999-2014. Investigación económica, 77(305), 74-96. doi: 10.22201/fe.01851667p.2018.305.67484

Elhorst, J. P. (2014). Spatial Econometrics from Cross-Sectional Data to Spatial Panels. Germany, Springer-Verlag Berlin Heidelberg.

Engen, E., \& Skinner, J. (1992). Fiscal policy and economic growth. (NBER, Working Paper No. 4223). doi: 10.3386/w4223

Erquizio, A., \& Ramírez R. (2014). La Recesión 2009 y la expansión 2010-2012 en las entidades federativas de México. Estudios Fronterizos. Revista de Ciencias Sociales y Humanidades, 15(30), 181-213. Recuperado de http://www.scielo.org.mx/scielo.php?script=sci_arttext\&pid=S0187$69612014000200007 \& \operatorname{lng}=\mathrm{es} \& \operatorname{tlng}=\mathrm{es}$

Fonseca, F. (2009). El impacto de la inversión pública sobre la inversión privada en México. Estudios Económicos, 24(2), 187-224. doi: 10.24201/ee.v24i2.125

Fuentes, N. A., \& Mendoza, E. (2003). Infraestructura pública y convergencia regional en México, 1980-1998. Comercio Exterior, 53(2), 178-187. Recuperado de: http://revistas.bancomext.gob.mx/rce/magazines/15/8/fuen0203.pdf

Fuentes, N. A. (2003). Crecimiento económico y desigualdades regionales en México: el impacto de la infraestructura. Región y Sociedad, 15(27), 81-103. doi: 10.22198/rys.2003.27.a658

Greenwald, B., \& Stiglitz, J. E. (1987). Keynesian, New Keynesian and New Classical Economics. Oxford Economic Papers, 39(1), 119-133. doi: 10.1093/ oxfordjournals.oep.a041773

Hansson, I. (1984). Marginal cost of public funds for different tax instruments and government expenditures. Scandinavian Journal of Economics, 86(2), 115130. doi:10.2307/3439685

Hernández, J. L. (2011). La relación gasto público-crecimiento en México, 19802009. Paradigma Económico, 3(2), 5-32. Recuperado de: https://paradigmaeconomico.uaemex.mx/article/view/4784

Hernández, Z., \& Velázquez, D. (2019). Políticas económicas estatales en México ante la Gran Recesión. En Z. Hernández, D. Velázquez \& H. Willebald (coord.), México y Uruguay ante la Gran Recesión, Pachuca de Soto: 
UAEH. Recuperado de: http://www.iecon.ccee.edu.uy/mexico-y-uruguay-ante-la-gran recesion/publicacion/747/es/

INEGI (2020). Sistema de Cuentas Nacionales de México. Producto Interno Bruto por Entidad Federativa. Año Base 2013. Serie de 2003 a 2018. Recuperado de https://www.inegi.org.mx/programas/pibent/2013/

Kim, M. (2019). Cross-industry distribution of R\&D investments and economic growth. Applied Economics Letters, 1(6), 163-167. doi: 10.1080/13504851.2019.1644426

King, R. G., \& Rebelo, S. (1990). Public policy and economic growth: developing neoclassical implications. Journal of Political Economy, 98(5), S126-S150. doi: $10.1086 / 261727$

Krugman, P. (1991). Increasing Returns and Economic Geography. Journal of Political Economy, 99(3), 483-499. doi: org/10.1086/261763

Krugman, P., \& Venables, A. J. (1995). Globalization and the Inequality of Nations. The Quarterly Journal of Economics, 110(4), 857-880. doi: org/10.2307/2946642

Lambarri, A. (2021). Cuando las prioridades son otras. IMCO. Centro de Investigación en Política Pública. Recuperado de https://imco.org.mx/cuandolas-prioridades-son-otras/

Lindbeck, A. (1983). Budget expansion and cost inflation. American Economic Review, 73(2), 285-296. Recuperado de: https://www.jstor.org/ stable $/ 1816856$ ?seq $=1$

Loría, E., Moreno-Brid, J. C., Salas, E., \& Sánchez-Juárez, I. (2019). Explicación kaldoriana del bajo crecimiento económico en México. Problemas del desarrollo, 50(196), 3-26. doi: org/10.22201/iiec.20078951e.2019.196.63506

Lopez-Bazo, E., Vaya, E., \& Artis, M. (2004). Regional Externalities And Growth: Evidence From European Regions. Journal of Regional Science, 44(1), 43-73. doi: 10.1111/j.1085-9489.2004.00327.x

Lustig, N. (2002). México hacia la reconstrucción de una economía. México: Fondo de Cultura Económica.

Mankiw, G. N. (1989). Real business cycles: A new Keynesian perspective. The Journal of Economic Perspectives, 3(3), 79-90. doi: 10.1007/978-1-349-24002-9_18

Marjit, S., Sasmal, R., \& Sasmal, J. (2020). Composition of public expenditure and growth of per capita income in Indian states: a political perspective. Journal of Social and Economic Development, 22(1), 1-17. doi: 10.1007/ s40847-020-00097-y

Mejía, P., \& Vergara, R. (2017). Are more severe recessions followed by stronger early expansions of employment in the Mexican states? The Review of Regional Studies, 47(3), 243-269. Recuperado de https://ideas.repec.org/a/ rre/publsh/v47y2017i3p243-269.html 
Mejía, P., Reyes M. R., \& Sánchez K. (2017). Gasto público y crecimiento económico de los estados del centro de México: un análisis de cambio estructural para el periodo 1980-2012. Paradigma Económico, 9(2), 33-64. Recuperado de: https://paradigmaeconomico.uaemex.mx/article/view/9390 Mejía, P., Rendón, L., Vergara, R., \& Aroca, P. (2018). International synchronization of the Mexican states business cycles: explaining factors, North American Journal of Economics and Finance, 44, 278-288. doi: org/10.1016/j. najef.2018.01.009

Mejía, P., \& Sánchez, G. (2019). Ciclos económicos y gasto público: un análisis de cambio estructural para la región centro de México, 1980-2015. Paradigma Económico, 11(2), 5-38. doi: 10.36677/paradigmaeconomico.v11i2.10064

Mendoza, M. Á., \& Valdivia, M. (2016). Remesas, crecimiento y convergencia regional en México: aproximación con un modelo panel-espacial. Estudios Económicos, 31(1), 125-167. Recuperado de http://www.scielo.org.mx/scielo. php?script=sci_arttext\&pid=S0186-72022016000100125\&lng=es\&tlng=es.

Mendoza-Cota, J. E., \& Calderón, C. (2006). Impactos regionales de las remesas en el crecimiento económico de México. Papeles de población, 12(50), 197221. Recuperado de: http://www.scielo.org.mx/scielo.php?script=sci_arttext\&pid=S1405-74252006000400010\&lng=es\&tlng=es.

Mendoza-Cota, J. E., \& Torres-Preciado, V. H. (2019). The impact of regional remittances on economic growth in Mexico: a dynamic space-time panel approach. Papeles de población, 25(101), 113-144. doi: org/10.22185/244 87147.2019.101.25

Meyer, D., \& Shera, A. (2017). The impact of remittances on economic growth: An econometric model. Economía, 18(2), 147-155. doi: org/10.1016/j. econ.2016.06.001

Moreno-Brid, J. C., \& Ros, J. (2009). Development and Growth in the Mexican Economy: An Historical Perspective. Oxford: Oxford University Press. doi:10.1093/acprof:oso/9780195371161.001.1

Noriega, A., \& Fontenla, M. (2007). La infraestructura y el crecimiento económico en México. El Trimestre Económico, 74(296), 885-900. doi: 10.20430/ete. v74i296.386

Nworji, I. D., Okwn, A. T. Obiwurn, T. C. \& Nworji, L. O. (2012). Effects of public expenditure on economic growth in Nigeria: a disaggregated time series analysis. International. Journal of Management Sciences and Business Research, 1(7), 1-15. Recuperado de: http://www.ijmsbr.com/Volume \%20 1,Issue $\% 207 \% 20(6) \% 20$ Andy.pdf

Olugbenga, A., \& Owoye, O. (2007). Public expenditure and economic growth: New evidence from OECD countries. Business and Economic Journal, 
4(17), 13-25. Recuperado de: https://iaes.confex.com/iaes/Rome_67/techprogram/P3164.HTM

Pinilla, D. E., Jiménez, J. D., \& Montero, R. (2013). Gasto público y crecimiento económico. Un estudio empírico para América Latina. Cuadernos de economía, 32(59), 181-210. Recuperado de: https://www.redalyc.org/ pdf/2821/282126853009.pdf

Posada, C. E., \& Gómez W. (2002). Crecimiento económico y gasto público: un modelo para el caso colombiano. Ensayos Sobre Política Económica, 20(4142), 5-86. doi: 10.32468/Espe.41-4201

Puga, D., \& Venables, A. J. (1996). The Spread of Industry: Spatial Agglomeration in Economic Development. Journal of the Japanese and International Economies, 10(4). doi: 10.1006/jjie.1996.0025

Rendón, L., Mejía, P., \& Vergara, R. (2019). Distribución espacial y especialización del empleo manufacturero de los municipios del centro de México, 19982013. Equilibrio Económico. Revista de Economía, Política y Sociedad, 15(47), 35-60. Recuperado de http://www.erevistas.uadec.mx/index.php/ $\mathrm{EE} /$ article/view/7

Rendón, L., Mejía, P., \& Díaz, M. (2021). Empleo manufacturero de los estados del centro de México. Análisis shift and share tradicional y con modificación de estructuras, 1998-2018. Paradigma Económico, 13(1), 5-34. doi: org/10.22136/est20191248

Reyes, M. R., y Mejía, P. (2016). Ciclo político presupuestal en México, 19802014. Un enfoque econométrico. Gestión y Política Pública, 25(2), 415445. Recuperado de http://www.scielo.org. $\mathrm{mx} / \mathrm{scielo}$.php?script=sci arttext\&pid=S1405-10792016000200415\&lng=es\&tlng=es.

Ros, J. (2015). ¿Cómo salir de la trampa del lento crecimiento y alta desigualdad? México: Colegio de México-UNAM. doi: 10.1016/j.inveco.2016.03.008

Sánchez, I., \& García, R. (2016). Public Debt, Public Investment and Economic Growth in Mexico. International Journal of Financial Studies, 4(2), 6. doi: org/10.3390/ijfs4020006

Sayan, S., (2006). Business cycles and workers' remittances: how do migrant workers respond to cyclical movements of GDP at home? (IMF, Working Paper, No. 2006/052). Recuperado de https://www.imf.org/external/pubs/ $\mathrm{ft} / \mathrm{wp} / 2006 / \mathrm{wp} 0652 . \mathrm{pdf}$

Semmler, A., Greiner, A., Diallo, B., Rajaram, A., \& Rezai A. (2007). Fiscal Policy, Public Expenditure Composition and Growth: Theory and Empirics (Policy Research Working Paper No. 4405). Recuperado de https://openknowledge. worldbank.org/handle/10986/7641 
Sorensen, P. B., \& Whitta-Jacobsen, H. J. (2010). Introducing advanced macroeconomics: growth and business cycles. Edimburgo: McGraw-Hill. doi: 10.5937/ekonhor1301097M

Srithongrung, A., \& Sánchez, I. (2015). Políticas fiscales y crecimiento económico subnacional en México, Revista Internacional de Economía y Asuntos Financieros, 5(1), 11-22. Recuperado de: https://www.econjournals.com/ index.php/ijefi/article/view/962/pdf

Turnovsky, S. J. (1996). Optimal tax, debt, and expenditure policies in a growing economy, Journal of Public Economics, 60(1), 21-44. doi: 10.1016/00472727(95)01519-1

WEF (2009). Mexico. The Global Competitiveness Index in detail. In The Global Competitiveness Report 2009-2010 (p. 223). Geneva, Switzerland: World Economic Forum.

WEF (2010). Mexico. The Global Competitiveness Index in detail. In The Global Competitiveness Report 2010-2011. (p. 239). Geneva, Switzerland: World Economic Forum.

WEF (2011). Mexico. The Global Competitiveness Index in detail. In The Global Competitiveness Report 2011-2012 (p. 259). Geneva, Switzerland: World Economic Forum.

WEF (2012). Mexico. The Global Competitiveness Index in detail. In The Global Competitiveness Report 2012-2013 (p. 257). Geneva, Switzerland: World Economic Forum.

WEF (2013). Mexico. The Global Competitiveness Index in detail. In The Global Competitiveness Report 2013-2014 (p. 277). Geneva, Switzerland: World Economic Forum.

WEF (2014). Mexico. The Global Competitiveness Index in detail. In The Global Competitiveness Report 2014-2015 (p. 271). Geneva, Switzerland: World Economic Forum.

WEF (2015). Mexico. The Global Competitiveness Index in detail. In The Global Competitiveness Report 2015-2016 (p. 259). Geneva, Switzerland: World Economic Forum.

(C) 2021 por los autores; licencia no exclusiva otorgada a la revista Estudios económicos. Este artículo es de acceso abierto y distribuido bajo los términos y condiciones de una licencia Atribución-No Comercial 4.0 Internacional (CC BY-NC 4.0) de Creative Commons. Para ver una copia de esta licencia, visite http://creativecommons.org/licenses/by-nc/4.0 
